\title{
A Systematic Evaluation of the Communicability of Online Privacy Mechanisms with Respect to Communication Privacy Management
}

\author{
Periambal L. Coopamootoo and Debi Ashenden \\ Department of Informatics \& Systems Engineering, \\ School of Defence \& Security \\ Cranfield University \\ Shrivenham, Swindon, UK, SN6 8LA \\ \{p.coopamootoo, d.m.ashenden\} @eranfield.ac.uk
}

\begin{abstract}
Online privacy mechanisms have not been effective in ensuring endusers' privacy. One of the main reasons is the un-usability of these mechanisms. Although past socio-psychological studies have highlighted the need for privacy in interpersonal interactions and social relationships, approaches to designing online privacy have often not considered privacy as a communication process. In this study the principles of communication privacy management (CPM) are used within semiotic inspection to examine online privacy mechanisms. We found that privacy as a communication process breaches many of the principles of CPM. We conclude that this might explain why end-users do not interact with online privacy mechanisms effectively.
\end{abstract}

\section{Introduction}

In the past few years, research has shown that although individuals claim to have high privacy concerns, they do not behave privately online [1]. Although online privacy mechanisms have been designed, they have not been very effective in ensuring endusers' privacy. A key reason is the un-usability of online privacy mechanisms. It is a challenging task to ensure the usability of online privacy mechanisms since privacy is part of an individual's communication behaviour and relationship building [2;3] and is not easily transposed into online functionality. Privacy is also person and context dependent. Although past socio-psychological studies have highlighted the need for privacy in interpersonal interactions and social relationships [4; 5], approaches to designing online privacy have not considered privacy as being an interpersonal communication process.

A study was performed with the aim of determining whether privacy mechanisms are designed in a way that mirror how individuals interact in an offline environment and hence whether they offer the functionality that end-users require. We analysed the online privacy mechanisms using a widely studied and applied theory of privacy communication - Communication Privacy Management (CPM) theory [6].

CPM is an evidenced-based theory of how individuals manage both their disclosures and those granted to them by others. CPM has been used in previous research to 
understand how people decide to disclose private information in offline settings and also to understand and address the tension between disclosure and privacy within the ecommerce context [7]. CPM offers a practical framework which can be used to assess online systems and consists of assumptions and interaction principles [8].

The analysis for this study was performed using the semiotic inspection method (SIM) which is a theory-based analytical usability evaluation method that concentrates on the communication aspects of HCI [9]. The purpose of theory-based evaluation methods in HCI is to assess the quality of interfaces and interactions in the light of a given perspective of HCI. The primary purpose of SIM is to evaluate the communicability of interactive computer artefacts by focusing on user interface meanings expressed through design. SIM helps to examine the diversity of signs presented to end-users while they interact with HCI artefacts and places the concept of the sign at the heart of semiotic inspection [10]. The signs present in computer interfaces include words, colours, dialogue structures and graphic layouts.

In this paper we describe the usability evaluation study undertaken to explore the communicability, and therefore the usability, of three cases of privacy mechanisms. Communicability is an important aspect of usability since not only can it contribute to the understanding required for learnability, memorability, ease of use, efficiency and satisfaction but also to the understanding of the privacy features which is an important part of exercising privacy online.

\section{Method}

In this section, the different types of privacy designs are first introduced, followed by a brief rationale behind the selection of particular examples that will be used for the communicability evaluation. The CPM principles are then explained.

\subsection{Privacy Designs}

Privacy designs can be categorised by the approach used to provide privacy, for instance, the 'notice and choice' type provides a privacy notice, which is usually a long sequence of text that acts as liability shield for the service provider, and choice for the end-user to decide whether to be private or to disclose [11]. Another type of privacy design is permission management which can be both embedded within the system's interaction design or added-on. A third type of privacy design is privacy protection (for example, through pseudonymity) where a trusted third-party provides end-users with a pseudonym that can be used to interact with the service provider so as not to reveal their full identity. In this study we selected an example of each of these privacy designs.

For the 'notice and choice' type, the Amazon web site was selected due to its wide use for e-commerce. For the privacy management type, the Internet Explorer 8 browser was selected since it is the most widely used internet browser. For the pseudonym type it was difficult to find a publicly available example. Finally, the Microsoft Dreamspark portal was selected for this type of privacy approach since it allows registered students access to services without the need to know who the students are. Moreover, verification of student status can be performed through the 
use of a student identity number or the use of Athens and Shibboleth institution logins. The Shibboleth scenario was used for the study. It is a standard based, web single sign-on package that can be used across, or within, organizational boundaries. It allows sites to make informed authorization decisions for individual access of protected online resources in a privacy-preserving manner [12].

\subsection{Communication Privacy Management Theory}

CPM theory was devised to understand the way people manage private information from a communication perspective targeting the privacy regulation that takes place during interpersonal interactions. It can be used to untangle the dialectical tension of both disclosing and maintaining privacy. In this study the theory was applied to the chosen designs to help understand and evaluate their usability. CPM theory is based on five principles of private information management that represent the organising principles interlinking both individuals and groups [13].

Principle One of CPM stipulates that disclosure and privacy form a dialectical tension; that is, if there is no disclosure, privacy mechanisms means are not required. When individuals disclose and they know they are disclosing, they usually communicate via some privacy rules.

Within Principle Two of CPM, individuals believe they own their personal information and hence have a perception of rightful ownership and a right to control it. They further believe that they are entitled to disclose information or keep it private depending on what seems the best choice for them. This leads to Principle Three of CPM, where it is stipulated that since individuals own their information and have a right to control it, they should have the means to regulate the flow of information that they define as private through the formation of privacy rules.

According to Principle Four, once private information has been disclosed, parties become responsible for co-owning and co-managing the information. This means that for a viable relationship to exist, and to allow smooth interaction between the parties involved in the disclosure, the rules must be coordinated through linkages. Linkages can be of different types depending on the balance of information shared between the parties and the balance of power that results.

Principle Five of CPM stipulates that dissimilar expectations and misunderstandings of privacy parameters can cause conflict in the handling of private information. This turbulence has to be dealt with by re-coordinating boundary rules.

It was decided that the use of SIM, bounded by CPM principles, would enable an understanding and evaluation of privacy mechanisms by teasing out the communication aspects of privacy within a systematic and rigorous analysis of the design. It has been argued that the interpretive results of SIM are objective, can be validated, and are comparable to other accepted methods because of the preparation and validation steps of the inspection method [9].

\subsection{Data Collection and Analysis}

SIM is systematically carried out in five steps. The first three steps consist of the inspection of documentation and 'Help' content together with the static interface signs and dynamic interaction signs. The questions asked at each of these steps refer to the 
principles of the CPM with the aim of understanding whether online privacy has been designed and communicated in a way that end-users are used to offline. The 'Help' content, static interface signs and dynamic interaction signs were then collated separately into three meta-communication templates.

For Amazon the 'Help' instances consisted of the privacy notice, the privacy paragraph in the 'Customer promise' and the help on the 'Your profile' section of the web site. The static interfaces selected were the 'Create your profile - your page on Amazon.co.uk' and the 'View basket' interface before proceeding to checkout. The pages 'Create a profile', 'Place an order' and 'Write a review' were chosen for the dynamic interactions.

For Internet Explorer 8, the 'Help' instances included 'Change your privacy settings', 'Customise your privacy settings for an individual website', 'Understanding privacy polices' and 'Understanding security and privacy features'. The static interface was the only interface presented by the privacy tab whereas dynamic interactions emerging from this interface included: selection of a setting for internet zone, customisation of privacy settings for an individual website (sites feature), selection of the advance option, selection of default and checking pop-up blocker followed by settings.

For Microsoft Dreamspark, the 'Help' documentation selected was the privacy notice whereas for the static interfaces, the initial home page (and the home page after login in with Windows Live Id) was selected. The dynamic interaction examined consisted of the sign-in feature which is followed by verification and by download since this sequence of actions is necessary to obtain access to services and since different parties are involved that impacts how personal details are treated.

In the fourth step of SIM the templates were compared to identify consistency and contradictions, whether gaps in one template were filled by another template and whether the signs mutually reinforced each other. In the last stage the findings from the comparison were analysed with respect to the CPM principles and a substantiated judgement was made of communicative problems that may prevent end-users from understanding that they are disclosing, that privacy means are available and how they can be used and hence from effectively using these online privacy mechanisms.

\section{Results}

In this section, an analysis is provided for each of the cases with respect to the CPM principles. We start with the 'notice and choice' scenario of Amazon, followed by IE 8 privacy management and end with Microsoft Dreamspark and pseudonymity.

\subsection{Amazon}

As outlined in section 2.1 above Amazon provides a 'notice and choice' privacy design. This means that notice is provided to customers in a privacy notice and endusers have a choice to disclose or to maintain their privacy according to their needs. Data was collected for each of the sub-questions for each CPM principle and the output is shown in the table below. 
Table 1. Amazon inspection

\begin{tabular}{|c|c|c|c|c|}
\hline \multirow{2}{*}{\multicolumn{2}{|c|}{ CPM Principles }} & \multicolumn{3}{|c|}{ Amazon } \\
\hline & & \multirow{2}{*}{\begin{tabular}{|l|} 
Help \\
$r$ \\
\end{tabular}} & Static & Dynamic: \\
\hline \multirow{3}{*}{ Principle 1} & What & & $4 \quad x$ & $\checkmark \times x$ \\
\hline & Wha & 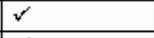 & $4 \quad x$ & $x$ \\
\hline & How & $r$ & $\checkmark x$ & $\checkmark \times \times$ \\
\hline \multirow{2}{*}{ Principle 2} & Choice to release or not & 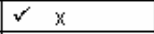 & $x$ & $x$ \\
\hline & Warning, notification & $\checkmark$ & $\checkmark x$ & $\checkmark \quad \times x$ \\
\hline \multirow{6}{*}{ Frinciple3 } & Means & $x$ & $x$ & $x$ \\
\hline & Amareness of means & $x$ & $x$ & $x$ \\
\hline & Motimation & $x$ & $x$ & $x$ \\
\hline & \begin{tabular}{|c|} 
Cantext \\
\end{tabular} & $r$ & $\psi$ & $r$ \\
\hline & Fisklbenefit & $x$ & $x$ & $x$ \\
\hline & Rule Acquire/social & $\begin{aligned} 4 \\
\end{aligned}$ & $x$ & $x$ \\
\hline \multirow{5}{*}{ Principle 4} & Who is linked & 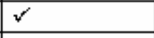 & $\begin{aligned} * \\
\end{aligned}$ & $\checkmark$ \\
\hline & Type of links & $\begin{array}{c}\text { role and } \\
\text { susceptibility }\end{array}$ & $\begin{array}{c}\text { coercive } \\
\text { and } \\
\text { identity }\end{array}$ & coercive \\
\hline & Amareness of type & \begin{tabular}{|l}
$r$ but not \\
for prafile
\end{tabular} & $\% \quad x$ & $x$ \\
\hline & Ownership & $\begin{array}{l}\text { manipulatiwe } \\
\text { and } \\
\text { obligatory }\end{array}$ & $\begin{array}{l}\text { obligatory' } \\
\text { with } \\
\text { amazon }\end{array}$ & manipulative \\
\hline & Permeability & $x$ & $x$ & $y$ \\
\hline \multirow{3}{*}{ Frinciple 5} & Awareness of turbulence & $x$ & $x$ & 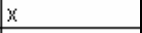 \\
\hline & Feedback after disclosure & $x$ & $x$ & $x$ \\
\hline & Means to deal with turbulence & $x$ & $x$ & $x$ \\
\hline
\end{tabular}

From the table we can see that for Principle One, Amazon allows the end-user to be aware, and to understand, that there will be disclosure of personal information. However, the static interface provides for awareness at the time of profile creation only and even then the recipient of disclosure is general. For the dynamic interaction sequences, the end-user would know that he or she would disclose personal information when creating a profile and have a general idea to whom but will not know this at a later stage when placing an order or writing a review.

For Principle Two, the above table shows that the 'Help' templates provide information that can reveal the release of control but not of ownership. 'Help' provides information about a change in boundary through either a warning or notification whereas the static interface and dynamic interactions do so only at the point of profile creation. For profile creation across the three meta-communication templates, the end-user might know about the release of control in the 'Help' sections as there is a sentence that says, 'you can also set who gets to view it: everyone or just you' whereas in the static interface and dynamic interactions it says, 'it's up to you with whom you share what'. The 'share' word suggests control rather than release. This distinction shows an inconsistency rather than mutual reinforcement across the three meta-communication templates. Furthermore, the 'Help' link is not very evident since it is on the top right corner of the screen and the 'Need help' button is similarly far from where the user interaction happens.

For Principle Three, it can be seen that overall Amazon does not provide the means, in terms of privacy features or mechanisms, to allow end-users to regulate the flow of their disclosure, although it does provide features of information management. The only indication of a privacy feature is within profile editing where the profile can 
be set to be completely private or open to everyone. However, the interactions do not lead to the editing feature. Amazon, by providing a 'notice and choice' architecture, is consistent in not providing a means to regulate flow of disclosure apart from profile creation where the user is warned with 'it's up to you what you disclose to whom' but still there is no means of regulating disclosure by what is shared to whom. The only solution would be for the end-user to not give any information rather than giving away everything. Moreover, the created profile is automatically set to public, and end-user would not know that this is the case; unless he or she edits the profile. Even so the 'edit' button is on the far right of the screen and is not embedded in what is happening during profile creation.

For Principle Four, although the end-user might know who is linked within the new boundary, the types of linkages formed differ between the 'Help' function, the static interfaces and the dynamic interactions. The types of linkages in the 'Help' sections are role or susceptibility linkages whereas the static and dynamic templates are of a more coercive nature due to the lack of information that would enable understanding. Ownership of shared information through the three templates is either obligatory or manipulative in nature. Moreover, no permeability is negotiated. The lack of boundary coordination and negotiation causes end-users to unknowingly acquire Amazon rules within both the static interface and dynamic interactions. Also, since the types of links are not clear, the end-user would not really know what is shared with whom, hence cancelling out the information provided in support of Principle One.

Amazon is consistent throughout its templates in not providing for turbulence awareness and a means to deal with turbulence according to Principle Five of CPM.

\subsection{Internet Explorer 8}

Internet Explorer 8 as mentioned in section 2.1 is the most widely used internet browser. It provides privacy management features through its privacy tab by enabling control of access to browsing activity information that can be used to profile endusers. The table below shows the data collected for the SIM analysis for each of the questions of CPM.

Table 2 shows that IE 8 does not communicate Principle One of CPM - that is awareness of disclosure - effectively to end-users. The 'Help' section clearly provides information about what is shared but is limited to a few examples whereas the static and dynamic templates are confusing with regards to what is shared to whom and how.

Table 2 also shows that IE 8 communicates Principle Two of CPM only through the 'Help' section and documentation. IE 8 does not effectively communicate awareness of the release of control and ownership which would have happened through a choice of control/ownership versus none and also does not communicate the awareness of changing boundaries which would happen through notifications or warnings.

In the 'Help' section, information about the means to regulate disclosure is available, but there is no information about risks versus benefits. However, the static interfaces and dynamic interactions provide no information that the features are a way 
of regulating disclosure. The static interfaces and dynamic interactions are also consistent in not providing motivation. The absence of motivation was detected by a lack of persuasive techniques compared to the 'Help' sections which provide for some motivation via suggestion, reduction and tunnelling techniques. They also provide no relation to the context of disclosure; do not enable a risk/benefit assessment and do not provide granular options that would favour formation of one's own rule combination.

Table 2. Internet Explorer 8 Inspection

\begin{tabular}{|c|c|c|c|c|}
\hline \multicolumn{2}{|c|}{ CPM Principles } & \multicolumn{3}{|c|}{ IE } \\
\hline & & $\mathrm{Help}$ & Static: & Dynamic \\
\hline \multirow{3}{*}{ Principle 1} & What & $\checkmark$ & $x$ & $x$ \\
\hline & Who & $x$ & $x$ & $x$ \\
\hline & How & $x$ & $x$ & $x$ \\
\hline \multirow{2}{*}{ Principle 2} & Choice to release or not & $\checkmark$ & $x$ & $x$ \\
\hline & Wivarning, notification & $\checkmark$ & $x$ & $x$ \\
\hline \multirow{6}{*}{ Principle3 } & Means & $\checkmark$ & $\gamma^{\prime}$ & $\psi^{\prime}$ \\
\hline & Awareness of means & $\checkmark$ & $x$ & $x$ \\
\hline & Motimation & $\checkmark$ & $x$ & $x$ \\
\hline & Context & $\checkmark$ & $x$ & $x$ \\
\hline & Fiskbenefit & $x$ & $x$ & $x$ \\
\hline & Fule Acouire/social & & & \\
\hline \multirow{5}{*}{ Principle 4} & Who is linked & $\checkmark$ & $\gamma^{\prime}$ & $\checkmark$ \\
\hline & Type of links & \multicolumn{3}{|c|}{ Role if user understands } \\
\hline & Awareness of type & $x$ & $x$ & $x$ \\
\hline & Owhership & $x$ & $x$ & $x$ \\
\hline & Permeability & $x$ & $x$ & $x$ \\
\hline \multirow{3}{*}{ Principle 5} & Awareness of turbulence & $x$ & $x$ & $x$ \\
\hline & Feedback after disclosure & $x$ & $x$ & $x$ \\
\hline & Means to deal with turbulence & $x$ & $x$ & $x$ \\
\hline
\end{tabular}

For Principle Four, the templates mention allowing or blocking cookies from 'first', 'third party' and 'browsing' websites. If end-users understand what cookies and these types of websites are, they might form role linkages; that is knowingly disclosing information to those with the role of providing a service and maintaining the disclosed information. However, the lack of explanation might result in end-users not understanding and so inadvertently agreeing to coercive linkages. There are also no ownership rights or permeability negotiations.

\subsection{Microsoft Dreamspark}

As explained in section 2.1, Microsoft Dreamspark portal was selected for the pseudonym type of privacy approach since it allows registered students access to its services through their pseudo-identity, that is, either through their registered student identity number or through a verified code from the student's academic institution. The verification scenario used for this study is Shibboleth authorisation. 
Table 3. Microsoft Dreamspark Inspection

\begin{tabular}{|c|c|c|c|c|}
\hline \multirow{2}{*}{\multicolumn{2}{|c|}{ CPM Principles }} & \multicolumn{3}{|c|}{ MS Dreamspark } \\
\hline & & $\mathrm{Help}$ & Static: & Dynamic \\
\hline \multirow{3}{*}{ Principle 1} & What & $\checkmark$ & $x$ & $x$ \\
\hline & W'Wo & $\checkmark$ & $x$ & $x$ \\
\hline & How & $\checkmark$ & $x$ & $x$ \\
\hline \multirow{2}{*}{ Frinciple 2} & Choice to release ar not & $x$ & $x$ & $x$ \\
\hline & Vvarning, notification & $x$ & $x$ & $x$ \\
\hline \multirow{6}{*}{ Principle3 } & Means & $\mathrm{x}$ & $x$ & $x$ \\
\hline & Awareness of means & $x$ & $x$ & $x$ \\
\hline & \begin{tabular}{|l|} 
Motivation \\
\end{tabular} & $x$ & $x$ & $x$ \\
\hline & Context & \multicolumn{3}{|c|}{ Access to M/S software } \\
\hline & Fisklbenefit & $x$ & $x$ & $x$ \\
\hline & Rule Acquire/social & $x$ & $x$ & $x$ \\
\hline \multirow{5}{*}{ Principle 4} & Who is linked & MS & MS & $\begin{array}{l}\text { MS } 8 \\
\text { Institution }\end{array}$ \\
\hline & Type of links & \multicolumn{3}{|c|}{ Role } \\
\hline & Awareness of type & $\checkmark$ & $x$ & $x$ \\
\hline & Owhership & $x$ & $x$ & $x$ \\
\hline & Permeability & $x$ & $x$ & $x$ \\
\hline \multirow{3}{*}{ Principle 5} & Awareness of turbulence & $\mathrm{x}$ & $x$ & $x$ \\
\hline & Feedback after disc:osure & $x$ & $\mathrm{x}$ & $x$ \\
\hline & Means to deal with turbulence & $\mathrm{x}$ & $x$ & $x$ \\
\hline
\end{tabular}

From Table 3 above, it can be seen that end-users will only understand that they are disclosing from the privacy statement and not through the static interface and the dynamic interactions. End-users will also only be aware of the type of links made from the privacy statement. The static interface and dynamic interaction sequence provides no other information about disclosure, privacy, the means to be private, boundary linkages, negotiation or turbulence. This analysis shows that this type of privacy-preserving approach controls personal details for the end-user but the enduser is not fully aware of who is involved in processing the information or how they can link personal details. For instance, it is unclear how much access Microsoft Dreamspark has to the end-user's institutional details. According to the technical aspects of Shibboleth, Microsoft would not have access but the end-user does not know that. Moreover, the amount of access that educational institutions have on the activities performed via Microsoft Dreamspark is not evident either.

\section{Discussion}

When the meta-communication templates from the 'Help' pages and privacy notice, the static interfaces and dynamic interactions are compared, the inconsistencies indicate that the release of meta-communication is incomplete by design, and such incompleteness contributes to end-users not understanding that they are disclosing to Amazon, IE 8 and Microsoft Dreamspark. If end-users cannot understand what they are disclosing, they cannot be expected to use privacy mechanisms and in Microsoft Dreamspark they are driven into using the mechanisms without being made aware of the fact. 
Moreover, while Amazon provides some indication of disclosure (Principle One) in its profile creation feature, the means to regulate disclosure (Principle Three) is not available. Hence the end-user does not have a choice. Furthermore, while Amazon indicates changing boundaries with a warning (Principle 2) for profile creation, it does not provide a choice of being private or disclosing. This means that although endusers would be aware that other customers will have access to their data, they would not realise that they are releasing control and ownership of that data.

Both Amazon and IE 8 provide quite blurred boundary linkage hints and do not allow for ownership and permeability negotiation. End-users might not even be aware how ownership of their data is shared - that is how much control the recipient has to act on his or her data. Turbulence awareness and resolution is also completely absent.

Amazon has a 'notice and choice' architecture that relies on its privacy notice to provide most of its privacy information to end-users but clear links to the 'Help' sections are not provided in the static interfaces and dynamic interactions. Furthermore the privacy notice could make use of a layered notice using icons for instance to enhance understanding. Amazon should also allow end-users to understand the risk and benefits of their actions.

Although IE 8 provides the means to regulate disclosure in the 'Help' section, it provides no awareness that these are privacy means or gives the motivation to use them or explains their relation to the context of disclosure in the static interfaces and dynamic interactions. The 'Help' section is not directly linked to the static interfaces and dynamic interactions. The consequence is that although the means are available, end-users would not know for what and how they can be used.

Finally, although Microsoft Dreamspark, through the use of Shibboleth, provides privacy to end-users by segregating institutional authorisation and software access, privacy communication is not performed by the end-user. The approach is easy to use, simple and usable, but it has taken control away from the end-users who will not necessarily understand who has access to their data and how. In this case the usability of the privacy mechanism has been reduced to functional usability through a technological approach. While this approach works in this scenario by keeping the end-user away from any decisions, it might not work for other scenarios. The current scenario also relies heavily on the privacy statement to inform end-users about the privacy of their details.

\section{Conclusion}

This study provides an evaluation of three different types of privacy approaches in terms of their interaction design and highlights the flaws in the way privacy awareness and control aspects are communicated to end-users. While a solution to making online privacy mechanisms more usable and effective is complex, this study helps to identify interface and interaction points that require enhancements. The designs that were analysed have not considered disclosure and privacy as a dialectical tension; the focus on disclosure and privacy are in different parts of the designs and they either give details of disclosure without providing the means to protect privacy or they provide privacy mechanisms without facilitating an awareness of what they 
offer. Moreover, the designs do not make end-users aware of how ownership rights are shared let alone assist them to decide those rights for themselves. Finally, endusers are only aware of permeability rights in certain instances but cannot negotiate these. This study then provides evidence that end-users cannot be expected to interact with these online privacy mechanisms effectively as the mechanisms offered do not map to the end-users' real-world expectations. The study forms part of an ongoing research on the usability of online privacy mechanisms.

\section{References}

1. Acquisti, A., Grossklags, J.: Losses, Gains, Hyperbolic discounting: an experimental approach to information security attitudes and behaviour. In: 2nd Annual Workshop on Economics and Information Security - WEIS 2003, May 29-30. University of Maryland (2003)

2. Gerstein, R.: Intimacy and Privacy. Ethics 89, 76-81 (1978)

3. Rachels, R.: Why Privacy is important? Philosophy of Public Affairs 4, 323-333 (1975)

4. Westin, A.: Privacy and Freedom. Athenum (1967)

5. Altman, I.: The Environment and Social Behaviour: Privacy, Personal Space, Territory and Crowding. Brooks/Cole Publishing, Monterey (1975)

6. Petronio, S.: Boundaries of privacy: dialectics of disclosure. State University of New York Press, Albany (2002)

7. Metzger, M.: Communication Privacy Management in Electronic Commerce. Journal of Computer-Mediated Communication 12(2) (June 2010), http://jcmc.indiana. edu/vol12/issue2/metzger.html

8. Petronio, S., Durham, W.T.: Communication Privacy Management Theory. In: Baxtor, L.A., Braithwaite, D.O. (eds.) Engaging Theories in Interpersonal Communication: Multiple Perspectives, pp. 309-322. Sage Publications, Inc., USA (2008)

9. de Souza, C.S., Leitao, C.F., Prates, R.O., Bim, S.A., da Silva, E.J.: Can inspection methods generate valid new knowledge in HCI? The case of semiotic inspection. International Journal of Human-Computer Studies 68, 22-40 (2010)

10. de Souza, C.S., Leitao, C.F., Prates, R.O., da Silva, E.J.: The Semiotic Inspection Method. In: VII Brazilian Symposium on Human Factors in Computing Systems, Natal, RN, Brazil, November 19-22, vol. 323. ACM, New York (2006)

11. Spiekermann, S., Cranor, L.: Engineering Privacy. IEEE Transactions on Software Engineering 35(1) (January 2009)

12. Morgan, R.L., Cantor, S., Carmody, S., Hoehn, W., Klingenstein, K.: Federated Security: The Shibboleth Approach. EDUCAUSE Quarterly 27(4), 31 (2011), http://www . educause.edu/EDUCAUSE+Quarterly/EDUCAUSEQuarterlyMagazineVol um/FederatedSecurityTheShibboleth/157315

13. Petronio, S.: Communication Privacy Management: What do we know about Family Privacy Regulation? Journal of Family Theory and Review 2(3), 175-195 (2010) 